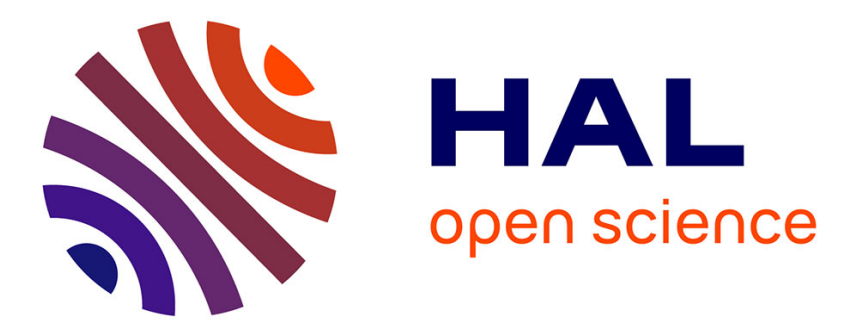

\title{
Magnetic Pulse Forming: Simulation and Experiments for high-speed forming processes
}

José-Rodolfo Alves Zapata, François Bay

\section{To cite this version:}

José-Rodolfo Alves Zapata, François Bay. Magnetic Pulse Forming: Simulation and Experiments for high-speed forming processes. AMPT 2014, Nov 2014, Dubaï, United Arab Emirates. hal-01112978

HAL Id: hal-01112978

https://hal-mines-paristech.archives-ouvertes.fr/hal-01112978

Submitted on 4 Feb 2015

HAL is a multi-disciplinary open access archive for the deposit and dissemination of scientific research documents, whether they are published or not. The documents may come from teaching and research institutions in France or abroad, or from public or private research centers.
L'archive ouverte pluridisciplinaire HAL, est destinée au dépôt et à la diffusion de documents scientifiques de niveau recherche, publiés ou non, émanant des établissements d'enseignement et de recherche français ou étrangers, des laboratoires publics ou privés. 


\title{
Magnetic Pulse Forming: Simulation and Experiments for high-speed forming processes
}

\author{
José R. Alves Z., a , François Bay ${ }^{1, b}$ \\ ${ }^{1}$ MINES ParisTech, PSL - Research University, CEMEF - Center for Material Forming - CNRS \\ UMR 7635 CS10207, F-06904 Sophia-Antipolis Cedex, France

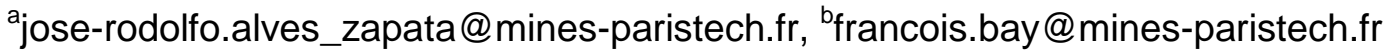

Keywords: Electromagnetic Forming, Finite Elements, Image analysis.

\begin{abstract}
In this paper we review the latest developments on high-speed forming at the Centre for Material Forming (CEMEF). We have developed a 3D Finite Elements analysis toolbox for the simulation of Electromagnetic forming (EMF) within the frame of the software FORGE. At the same time we have recently acquired an EMF machine to study the behavior of materials at high deformation speeds. We describe the modeling strategy for the simulation package together with some results for a ring expansion case. We also present the experimental settings and some preliminary results for a direct free forming of flat metal sheet.
\end{abstract}

\section{Introduction}

Interest in lightweight structural applications has driven research on alternative forming processes that allow costs reduction while maintaining or improving structural resistance. Electromagnetic forming (EMF) is among the techniques experiencing a rapid growth since highspeed forming allows increased formability properties beyond the limits encountered in quasi-static forming process [1].

A summary of the main advantages of EMF is given in [2]: Absence of mechanical contact between tool and work piece implies less surface deterioration and no need for lubrication. Joining of dissimilar materials becomes possible and reliable because a strong mechanical joint is created by impact, instead of a thermo-chemical reaction. Almost complete elimination of elastic springback is achieved, translating into simplification of die design. Formability is reported to be better at high strain rates and EMF allows strain rates in the order of $\left[10^{3}-10^{4}\right] \mathrm{s}^{-1}$.

EMF enables forming and joining processes in reduced spans of time $(200 \mu \mathrm{sec})$ through use of intense magnetic fields on an electrical conductive work piece (or driver). The induced currents produce repulsive Lorentz body forces on the work piece that accelerates it away from the tool coil. Forming takes place by the later transformation of the kinetic energy into plastic deformation [3] during either free deceleration or impact for joining or die forming processes.

Full understanding of the process is quite challenging because of the multiple physical aspects involved: namely, the electromagnetic wave propagation, fast induction heating and self-heating through deformation work, the inertial effects on the formability, etc. Hence the increased interest in developing numerical tools that provides accurate representation of the complete process. 


\section{Simulation Software}

We have developed a numerical toolbox based on Finite Elements methods for the analysis of electromagnetic forming applications. This toolbox is a coupling between the software tool FORGE - for coupled thermo-mechanical (TM) modeling of large material deformation - and MATELEC which solves the electromagnetic (EM) wave propagation problem based on Maxwell equations. The latter was developed for applications in induction heating and it is now extended to electromagnetic forming applications.

\subsection{Electromagnetic model}

The electromagnetic wave propagation phenomena is described by the well-known set of Maxwell's equations

$$
\begin{array}{ccc}
\nabla \times \vec{E}=-\partial_{t} \vec{B} & (\text { Eq.1 }) & \nabla \cdot \vec{D}=\rho^{e} \\
\nabla \times \vec{H}=\partial_{t} \vec{D}+\vec{J} & (\text { Eq.3 }) & \nabla \cdot \vec{B}=0
\end{array}
$$

Where $\vec{E}$ represents the electric field, $\vec{D}$ the electric flux density, $\vec{H}$ the magnetic field, $\vec{B}$ the magnetic flux density, $\vec{J}$ the electric current density, and $\rho^{e}$ the electric charge density. For many numerical applications it is more convenient to express this system into the potential formulation $(\phi, \vec{A})$ given by

$$
\begin{gathered}
\varepsilon_{0} \varepsilon_{r} \partial_{t}{ }^{2} \vec{A}+\sigma \partial_{t} \vec{A}+\frac{1}{\mu_{0}}\left(\nabla \times \frac{1}{\mu_{r}} \nabla \times \vec{A}\right)=-\sigma \nabla \phi-\varepsilon_{0} \varepsilon_{r} \partial_{t} \nabla \phi \\
\nabla \cdot(\sigma \nabla \phi)=0
\end{gathered}
$$

Where, $\sigma$ is the material electric conductivity, $\mu$ the magnetic permeability, $\varepsilon$ the electrical permittivity, subscripts " 0 " represents the value for void and " $r$ " the relative value with respect to the void. Additionally,

$$
\begin{array}{clc}
\vec{B}=\nabla \times \vec{A} & (\text { Eq.7 ) } & \vec{E}=-\nabla \phi-\partial_{t} \vec{A} \\
\vec{B}=\mu_{0} \mu_{r} \vec{H} & \text { (Eq.9) } & \vec{D}=\varepsilon_{0} \varepsilon_{r} \vec{E} \\
\vec{J}=\sigma \vec{E} & \text { (Eq.11) }
\end{array}
$$

Equation (5) is discretized in space using Nedelec elements [4]. Uniqueness of the solution is guaranteed through the implicit fulfillment of the gauge condition $\nabla \cdot \vec{A}=0$. The variational form is written as

$$
\begin{aligned}
& \varepsilon_{0}\left\langle\varepsilon_{r} \vec{W}, \vec{W}\right\rangle \partial_{t}{ }^{2}\{A\}+\langle\sigma \vec{W}, \vec{W}\rangle \partial_{t}\{A\} \frac{1}{\mu_{0}}\left\langle\frac{1}{\mu_{r}} \nabla \times \vec{W}, \nabla \times \vec{W}\right\rangle\{A\}= \\
&-\langle\sigma \nabla \varphi, \vec{W}\rangle\{\phi\}-\varepsilon_{0}\left\langle\varepsilon_{r} \nabla \varphi, \vec{W}\right\rangle \partial_{t}\{\phi\} \\
&\langle\sigma \nabla \varphi, \nabla \varphi\rangle\langle\phi\}=0
\end{aligned}
$$

Where $\{A\}$ and $\{\phi\}$ represent the set of edge values and nodal values respectively.

\subsection{Thermo-mechanical model}

Resolution of the TM problem starts with the well-known equations 
- Momentum equation

$$
\nabla \cdot \underline{\underline{\sigma}}+\vec{b}=\rho D_{t} \vec{v}
$$

Where $\underline{\underline{\sigma}}$ is the Cauchy stress tensor, $\vec{b}$ represent any volumetric force such as gravity (which is not taken into account in our analysis) or the Lorentz body forces, in which case

$$
\vec{b}=\vec{F}_{\text {lorentz }}=\vec{J} \times \vec{B}
$$

The inertia is described by the material density $\rho$ and the material time derivative of the velocity $D_{t} \vec{v}$.

- Energy equation

$$
\begin{gathered}
\rho d_{t} e=\rho C_{p} d_{t} T=-\nabla \cdot \vec{q}+\rho Q+\underline{\underline{\sigma}}: \underline{\underline{\dot{\varepsilon}}} \\
\rho C_{p} d_{t} T+\nabla \cdot(k \nabla T)=\rho Q+\underline{\underline{\sigma}}: \underline{\underline{\dot{\varepsilon}}}
\end{gathered}
$$

In equation (17) $C_{p}$ denotes the heat capacity, the main unknown $T$ the temperature, $k$ accounts for the heat conduction within the body, $Q$ describes any volumetric heat source, for instance the joule heat term given by

$$
Q_{\text {joule }}=\vec{J} \cdot \vec{E}
$$

and last, the self-heating due to the internal work $\underline{\underline{\sigma}}: \underline{\dot{\varepsilon}}$ where $\underset{\underline{\dot{\varepsilon}}}{\underline{\dot{\varepsilon}}}$ represents the strain-rate tensor. A full account of the assumptions and modeling techniques behind the TM problem is beyond the scope of the current work. We refer the interested reader to [5] for a detailed description of the finite element modelling for the metal forming processes, and to [6] for the modelling of the heat transfer phenomena in FORGE.

For the mechanical behaviour of the material it is selected to use an elasto-viscoplastic characterisation. The elasticity is described by the classic Young modulus $E$ and the Poisson coefficient $v$. The evolution of the flow stress is followed by means of the empirical Johnson-Cook model [7].

$$
\sigma_{e q}=\left(A+B \bar{\varepsilon}_{p l}^{n}\right)\left(1+C \ln \left(\frac{\dot{\varepsilon}_{p l}}{\dot{\varepsilon}_{0}}\right)\left(1-\left(\frac{T-T_{0}}{T_{m}-T_{0}}\right)^{m}\right)\right.
$$

Where $\sigma_{e q}$ denotes the effective stress, that for a isotropic solid is given by the von Mises equivalent, $\bar{\varepsilon}_{p l}$ is the effective plastic strain, $\dot{\varepsilon}_{p l}$ is the effective strain-rate, $\dot{\varepsilon}_{0}$ is a reference strainrate value, $T_{0}$ is the room temperature while $T_{m}$ represents the material melting point. $A, B, n, C$ and $m$ are the material constitutive parameters. 


\subsection{Methodology}

For the coupling of the TM and the EM problems we follow the general scheme shown next:

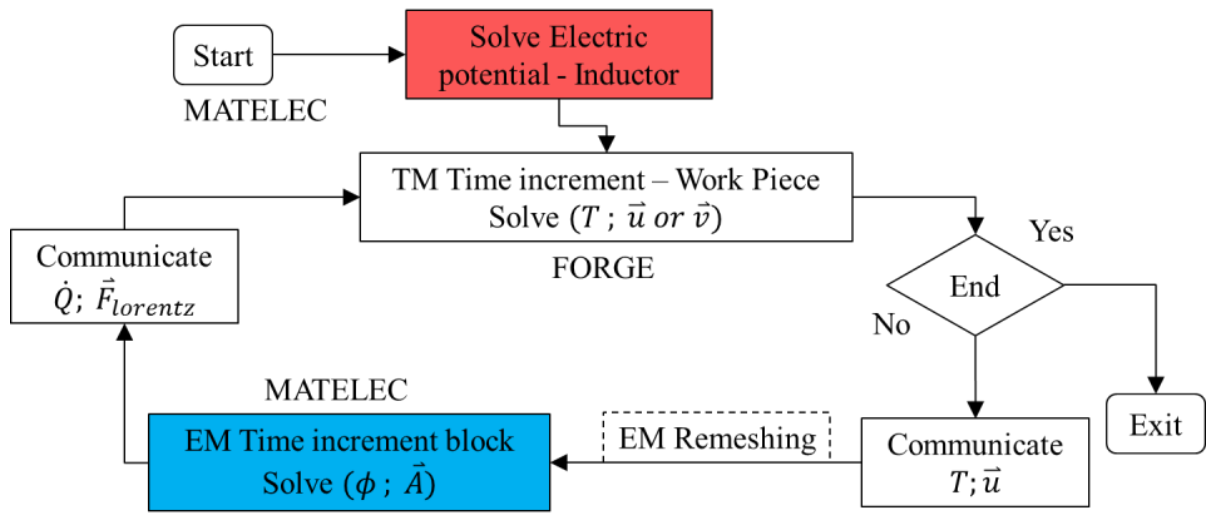

Figure 1 Global scheme of the coupling strategy between FORGE and MATELEC for EMF processes.

Each solver (MATELEC and FORGE) solves the physics in a separate way. At each time step, required data is transferred and updated for use in the next time step. This is known as a looselycoupled scheme.

Solving all the physics simultaneously in a single frame would be more accurate but also would be extremely expensive in computational terms. Also, the loosely-coupled approach permits adapting the meshes individually for each solver. As will be shown further, for the EM model we mesh the air surrounding the parts. A mesh adaptation method is implemented to allow the air following the movement of the solid parts.

\section{Validation: Analytical Model}

For the development and validation stage of the solver, it is recommended to choose a study case that can be easily analyzed through analytical formulations. Fenton [8] proposed the ring expansion experiment as the easiest model to perform plasticity characterization on metal specimens submitted to high speed loading.

It consists in two coaxial copper rings as in Figure 2. The inner ring serves as the inductor connected to the machine. It will receive the electric loading and generate the repulsive forces on the external ring, causing the latter to deform.

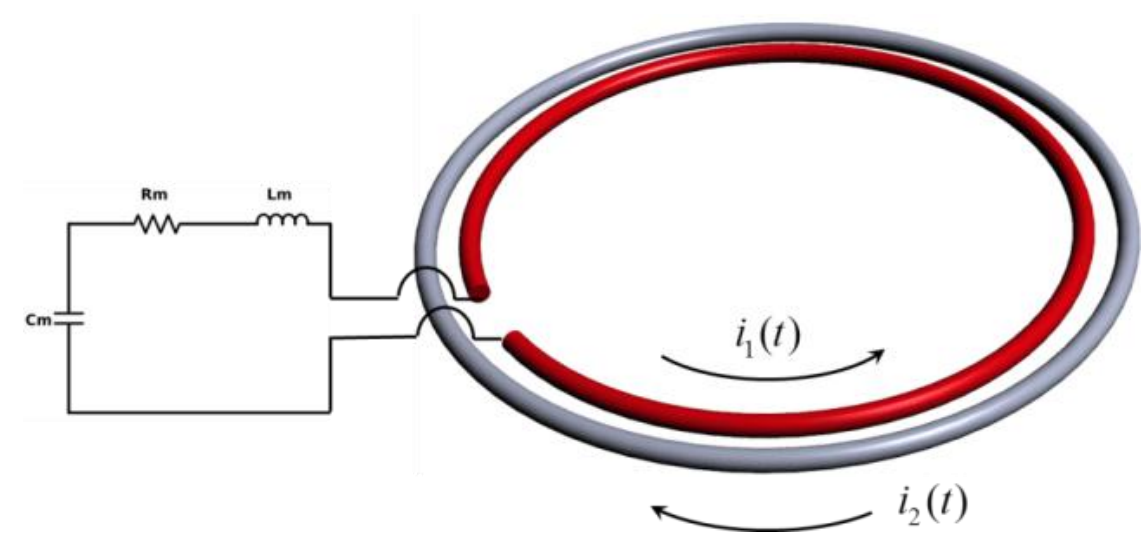

Figure 2 Ring expansion model configuration 
For the subsequent analysis, we will consider the following set of parameters

\begin{tabular}{lc}
\hline & Machine \\
\hline$V_{0}[$ Volts $]$ & 5000 \\
$C_{m}[\mu \mathrm{F}]$ & 126 \\
$R_{m}[\mathrm{~m} \Omega]$ & 48 \\
$L_{m}[\mathrm{nH}]$ & 115.7 \\
\hline
\end{tabular}

\begin{tabular}{ll}
\hline \multicolumn{2}{c}{ Geometry } \\
\hline Coil mean radius $a_{1}[\mathrm{~mm}]$ & 14 \\
Coil cross-section width $b_{1}[\mathrm{~mm}]$ & 1 \\
Ring mean radius $a_{2}[\mathrm{~mm}]$ & 16 \\
Ring cross-section width $b_{2}[\mathrm{~mm}]$ & 1 \\
\hline \multicolumn{2}{c}{ Table 2 Ring expansion case geometric parameters }
\end{tabular}

\begin{tabular}{|c|c|c|c|}
\hline \multicolumn{4}{|c|}{ Material: Copper } \\
\hline \multicolumn{2}{|l|}{ General } & \multicolumn{2}{|c|}{ Plasticity (Johnson-Cook Model) } \\
\hline Density $\rho[\mathrm{kg} / \mathrm{m} 3]$ & 8924 & $\mathrm{~A}[\mathrm{MPa}]$ & 90 \\
\hline \multicolumn{2}{|l|}{ Elastic } & $\mathrm{B}[\mathrm{MPa}]$ & 292 \\
\hline Young $E[\mathrm{MPa}]$ & $120 \mathrm{e} 3$ & $\mathrm{C}[-]$ & 0.025 \\
\hline Poisson $v[-]$ & 0.3 & $\mathrm{n}[-]$ & 0.31 \\
\hline \multicolumn{2}{|l|}{ Thermal } & $\mathrm{m}[-]$ & 1.09 \\
\hline \multirow{3}{*}{$\begin{array}{l}\text { Conductivity } k\left[\mathrm{~W} / \mathrm{m}^{*} \mathrm{~K}\right] \\
\text { Specific Heat } C_{p}\left[\mathrm{~J} / \mathrm{kg}^{*} \mathrm{~K}\right] \\
\text { Expansion } \alpha[1 / \mathrm{K}]\end{array}$} & 401 & $\operatorname{Tm}\left[{ }^{\circ} \mathrm{C}\right]$ & 1083 \\
\hline & 356 & \multicolumn{2}{|c|}{ Electric } \\
\hline & $16.6 \mathrm{e}-6$ & Resistivity $\rho^{e}[\Omega \mathrm{m}]$ & $1.68 \mathrm{e}-8$ \\
\hline
\end{tabular}

As we explained before, the simulation of the EMF process is carried out using separate models for the various physical problems involved. This requires also the creation of separate meshes for each simulation. For the electromagnetic simulation, it is necessary to take into account the two solids (inductor and work piece) and the surrounding air as shown in Figure 3.,For the TM simulation only the work piece is taken into account. This enables creating a mesh that is well adapted to the mechanical problem independently of the EM mesh. The necessary data will be interpolated from one mesh to the other.

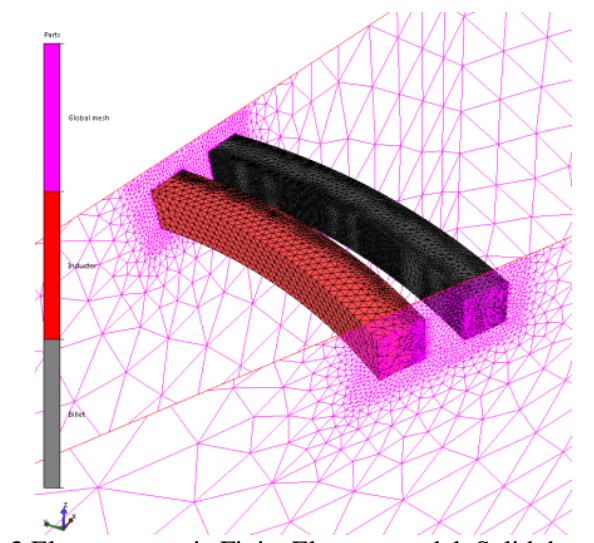

Figure 3 Electromagnetic Finite Element model. Solid domains are fully embedded within the air mesh.

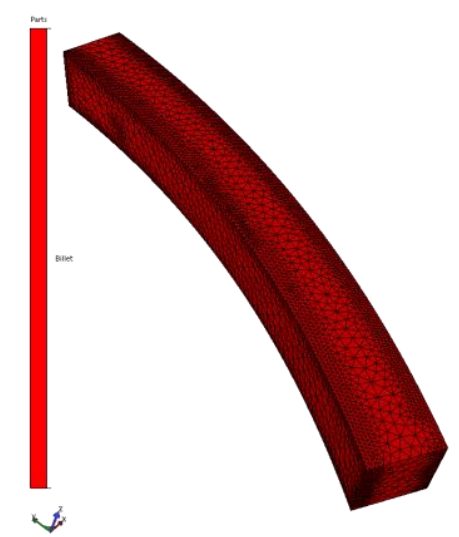

Figure 4 Thermo-mechanical Finite Element Model. Only the ring is simulated.

Within MATELEC we set-up all the electromagnetic properties of the study case plus the electric constants defining the machinery behind the process. Namely, the initial potential (energy level); the equivalent capacitance of the machine; and the equivalent resistance and inductance of the 
circuit connected from the machine to the coil. These four parameters are introduced as the driving values for the whole simulation.

In FORGE we define the thermo-mechanical properties: material behavior, contact (if a $3^{\text {rd }}$ part is used for impact analysis), thermal interactions and dissipation, etc.

\subsection{Electric Results}

One of the easiest observables in an EMF process is the resulting electric current of the system. By means of a Rogowski coil attached to the main circuit it is possible to capture the evolution of this global variable. For this reason we have selected the electric current to be one of the first observables from the simulation. In Figure 5 we compare the resulting electric current to the analytic solution (refer to [8] for details on the equations)., Globally the agreement between both is good, having just a 5\% difference on the peak value. Looking at the evolution of the discharge potential at the capacitor level Figure 6, the curves fit almost perfectly.

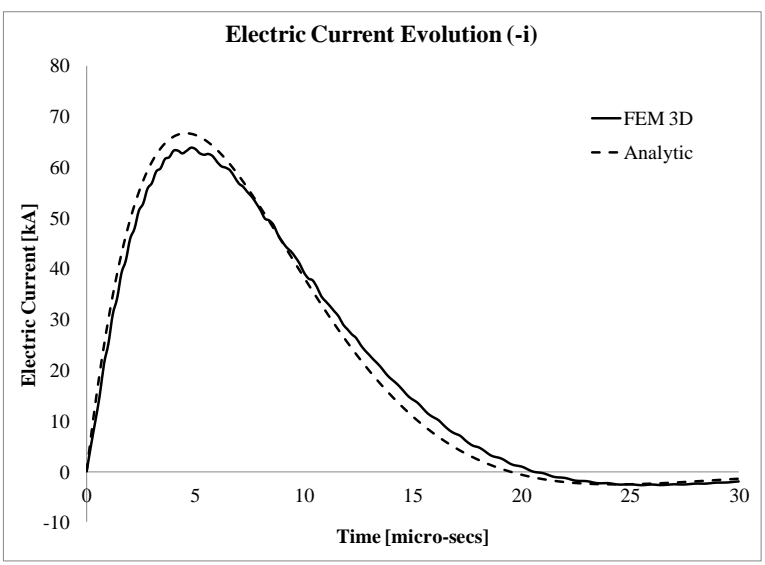

Figure 5 Comparison between the analytical model and the 3D FEM. Evolution of the resulting electric current on the inductor.

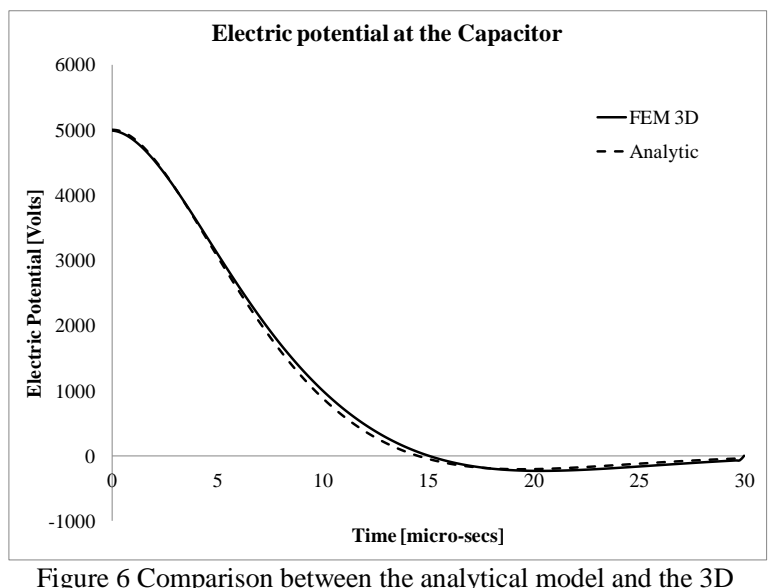

FEM. Evolution electric potential coming from the capacitor.

\subsection{Thermo-Mechanical Results}

As the main interest is to characterize the behavior of materials during the EMF process, we now take a look at the thermo-mechanical variables. For this purpose we have selected, as shown in Figure 7, 3 nodes and 3 elements at one of the symmetry faces of the model to read the output variables. The nodes are used to measure displacements, velocities and temperatures, the elements will provide the stress, strain, strain-rate and the Lorentz body forces. The latter are interpolated from the electromagnetic simulation. 


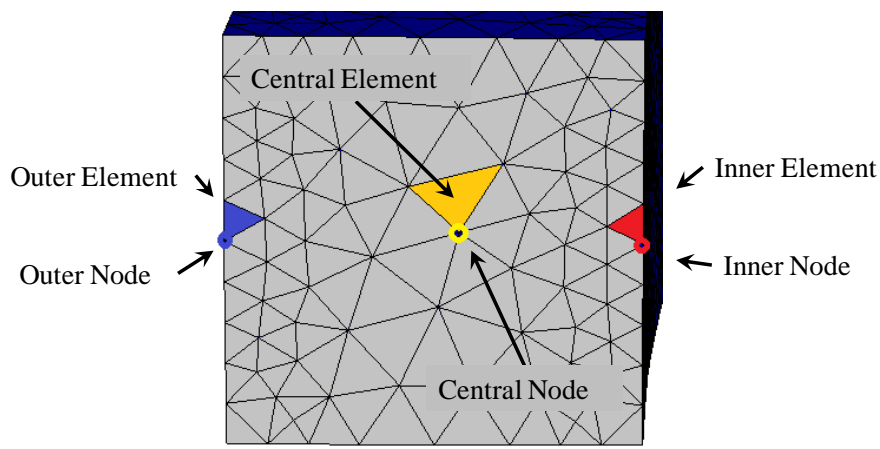

Figure 7 Selection of output positions. Inner, Central and Outer labels used for identification of the the variables with respect the ring radius.

Let us recall that from all the assumptions done for the analytical model, the most important one is the reduction to a $1 \mathrm{D}$ problem. All variables are considered uniform throughout the cross section of the solids. This permits focusing only on what happens at the center of the ring.

Observing the evolution of the kinematic variables we see that the analytic solution always overestimates the results (see Figure 8 and Figure 9). This result was already observed in the original work where they developed a $2 \mathrm{D}$ axisymmetric code to study the problem. On the other hand, it helps estimating the order of magnitude of the variables and the global behavior.

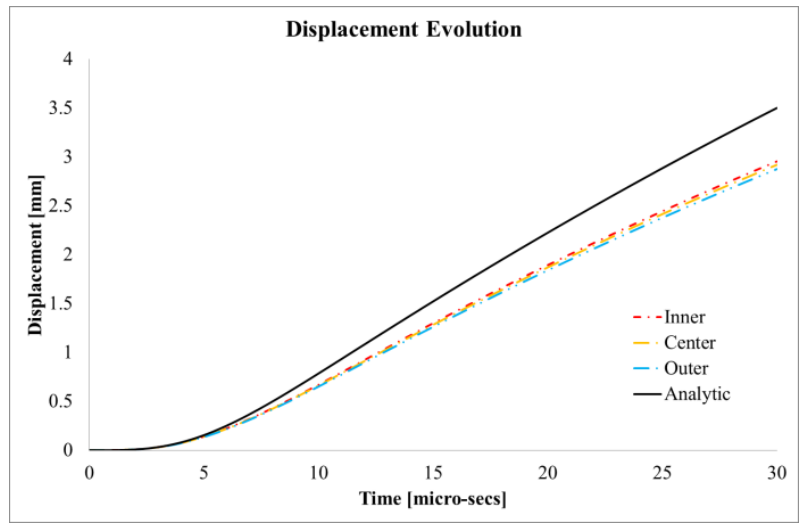

Figure 8 Evolution of the ring displacement. Comparison Analytic vs. FEM

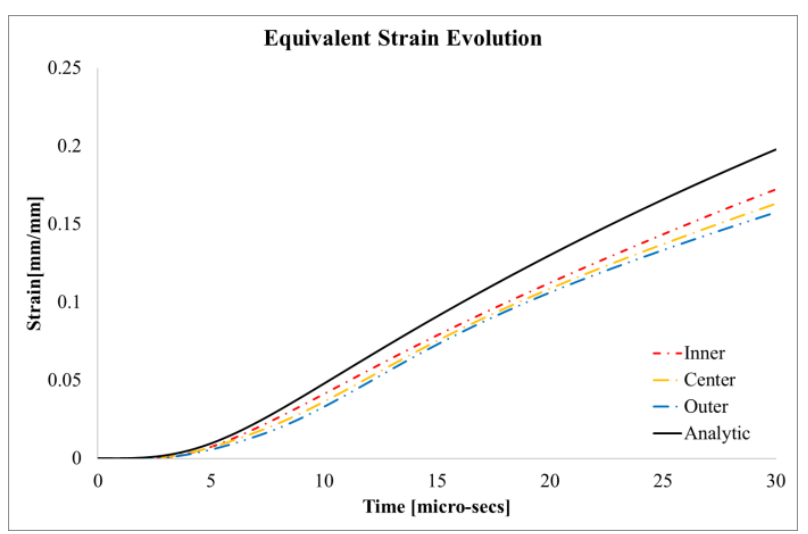

Figure 10 Evolution of the equivalent strain. Comparison Analytic vs. FEM

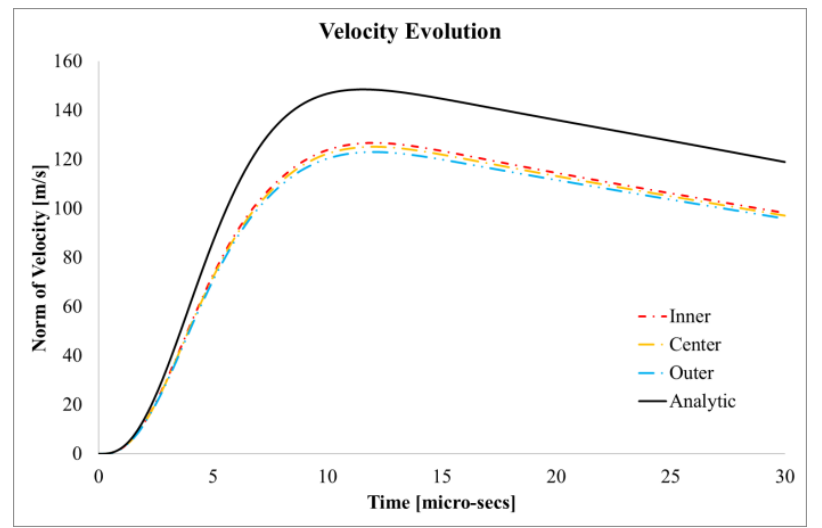

Figure 9 Evolution of the radial deformation speed. Comparison Analytic vs. FEM

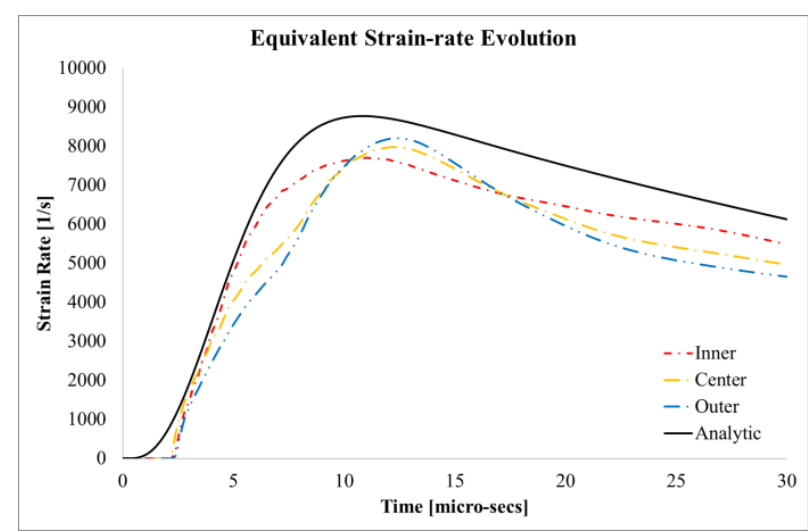

Figure 11 Evolution of the equivalent strain-rate. Comparison Analytic vs. FEM

The advantage of the finite element model resides in its ability to provide us with information on the local phenomena taking place during the forming process. When looking at the strain-rate for 
instance (Figure 11) it is found that the inner region withstands the largest strain-rates during most of the process, but the peak is actually found for the central and outer elements. This might be due to the transmission of the shock wave through the solid domain.

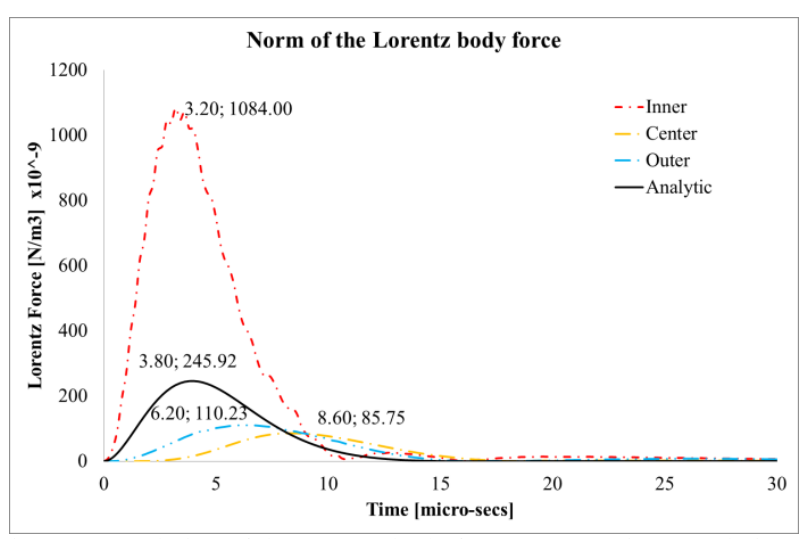

Figure 12 Evolution of the Lorentz body forces. Comparison Analytic vs.

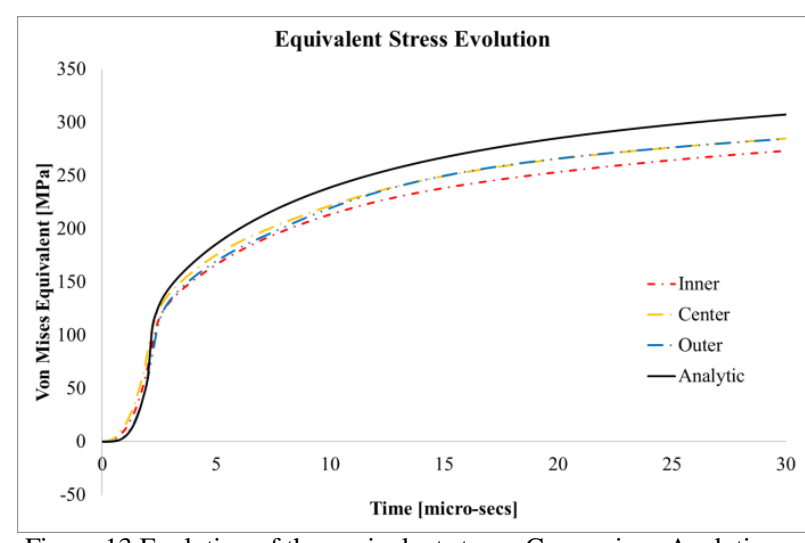

Figure 13 Evolution of the equivalent stress. Comparison Analytic vs.

Those results are in agreement with the stress levels observed in Figure 13. At the beginning of the process the stress level is basically homogeneous throughout the cross section (first 10micro seconds). Afterwards, the center and the outer elements read higher levels of stress compared with the inner element. Figure 12 reveals the lag in the transmission of the body forces. The inner layer (closer to the inductor) will concentrate the magnetic fields due to the skin effect. Nevertheless, the transmission of the wave causes the central and outer region to continue experiencing a higher level of forces while forces in the inner region remain close to zero.

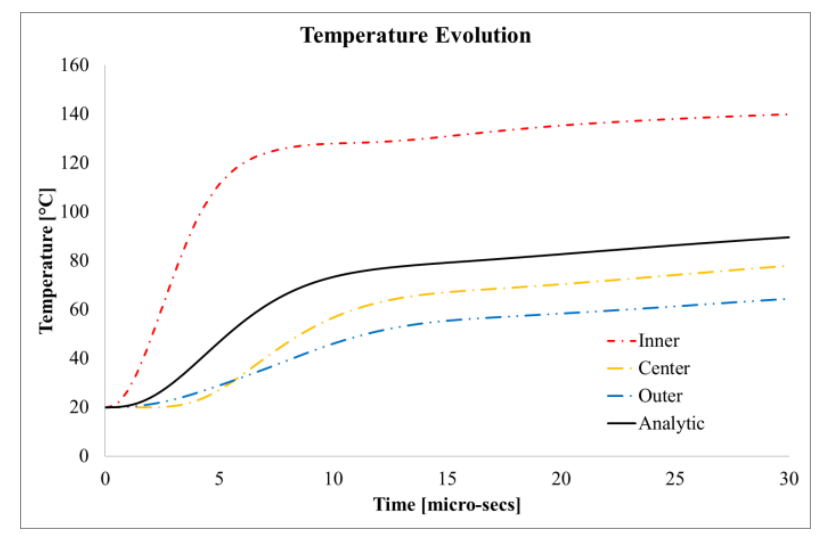

Figure 14 Evolution of the temperature increase due to self heating and joule heating. Analytic vs. FEM

The skin depth will also affect the temperature distribution in the body. The Joule heating effect at the skin causes a high increase in temperature locally. For this case we found $80^{\circ} \mathrm{C}$ difference between the outer and the inner nodes. Another interesting phenomenon revealed in Figure 14 is that during the first 5 micro-seconds the Outer temperature is higher than the center. This is due to the presence of the induced current mainly on the outer surfaces.

A 3D view of the fields distribution can be observed in Figure 15. We see how the Lorentz forces are highly concentrated in the inner region of the ring as well as on the surface of the coil. Since the Lorentz forces are directly proportional to the current density, it also helps confirming the previously shown results regarding the temperature distribution. 


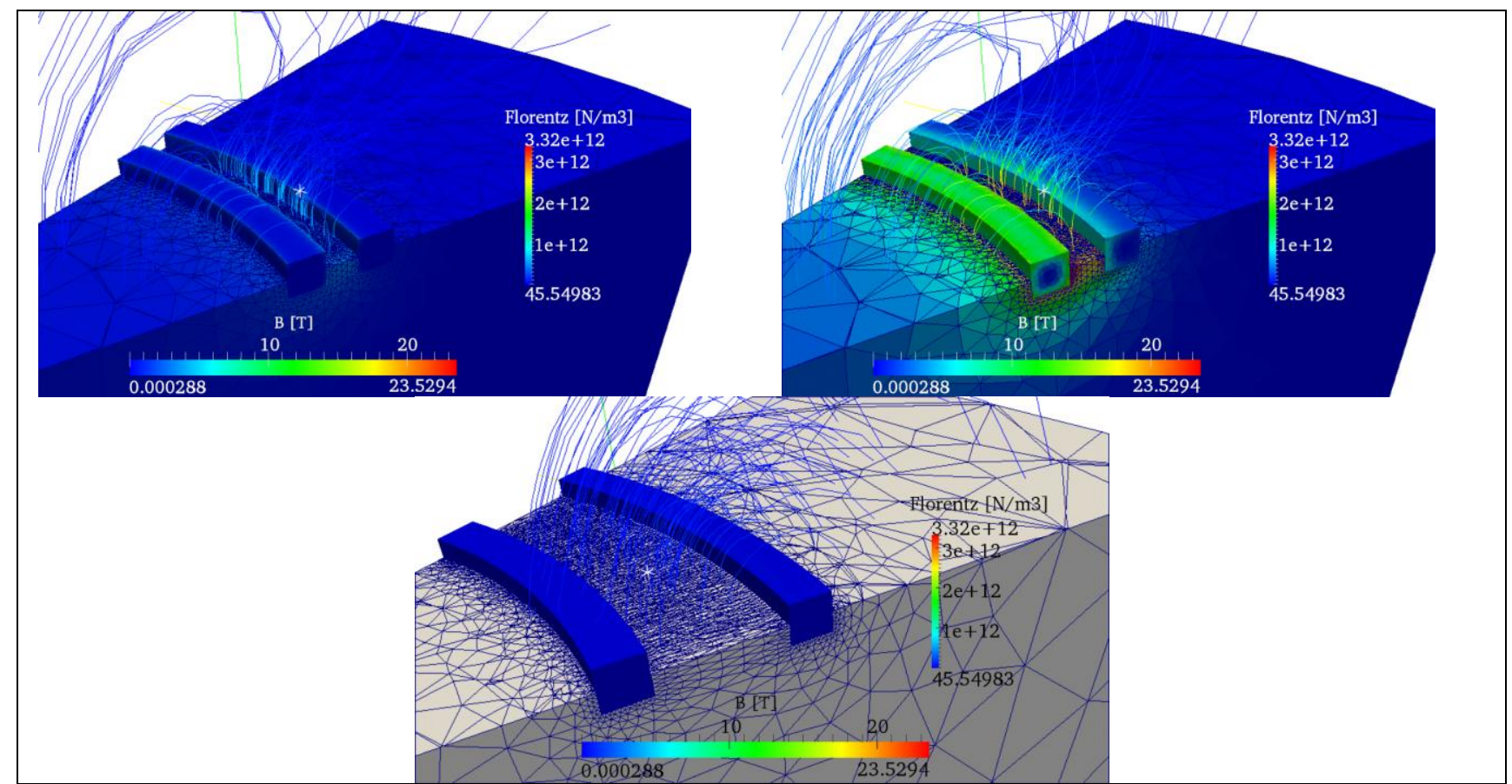

Figure 15 3D view of the electromagnetic model. On the solids: Lorentz force distribution. On the air: Magnetic field distribution. (Left) Fields at $\mathrm{t}=0.2$ micro-secs. (Right) Fields at $\mathrm{t}=2$ micro-secs. (Bottom) Fields at $\mathrm{t}=20$ micro-secs.

\section{Experimental Facilities}

So far we have presented the main developments related to the software for the simulation of the process. Nevertheless, any simulation result has to be confronted with experience in order to assess its validity. We have two main objectives

- Short term: Validate the software with complex cases.

- Medium and Long term: carry out complete studies on the behavior of materials at high deformation speeds.

For this purpose a EMF machine was acquired recently from BMAX industries, with whom we work in close collaboration. It enables achieving top energies of $25 \mathrm{~kJ}$ at $9 \mathrm{kVolts}$. The fast discharge of the large amount of energy will let us reach the desired deformation rates over $1000 s^{-1}$. 


\subsection{Machinery \& Experimental Set-up}

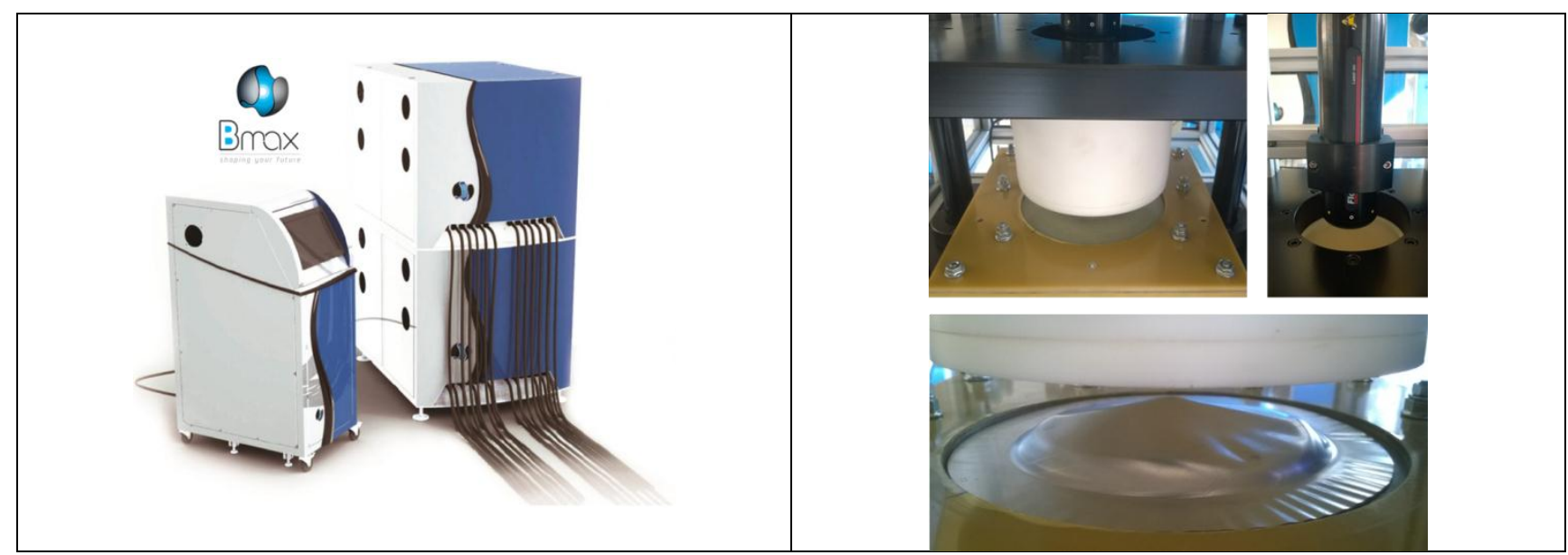

Figure 16 ElectroMagnetic Forming machine at CEMEF laboratories. (Left) Command box \& Capacitor bank. (Right) Work bench for free forming experiments.

The machine consists of three main modules. The control module and the capacitors bank are shown in Figure 16 (left). Here we set up desired potential and if necessary, it is possible to modify the capacitance in order to vary the discharge frequency of the system.

The third module is the work bench (Figure 16, right). We have adapted it for Direct Free Forming of flat metal sheets because of the complexities that can be observed in this type of processes. It consists in a 'Pancake' type coil on the top of which is placed the metal sheet. An epoxy layer is placed in between to avoid direct contact of the two solids as shown in Figure 17.

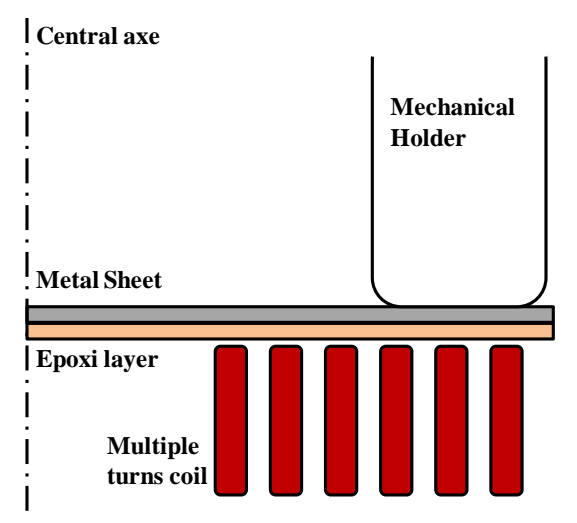

Figure 17 Set-up scheme of the work bench for EMF.

\subsection{Preliminary Results}

We have carried out several preliminary tests using 30x0.5 mm circular aluminum 1050a sheets for feasibility purposes. After several trials we have found out (results can be observed in Figure 18):

- Typical flange wrinkles observed in drawing processes appear for even low discharge potentials.

- The non perfect symmetry of the coil leaves a strong signature on the sheet as can be observed in the irregular formed surface. Anisotropy of the material due to the previous lamination process may well play an important role here.

- At $2.5 \mathrm{kV}$ olts we obtain a peak displacement in the center of the sheet of approximately $3 \mathrm{~cm}$. localized necking is already present in several points around the center. 
- For input potentials above $2.6 \mathrm{kV}$ olts we observe the beginning of fracture at the clamping zone due to the section thinning. At $3 \mathrm{kV}$ olts full fracture is obtained.

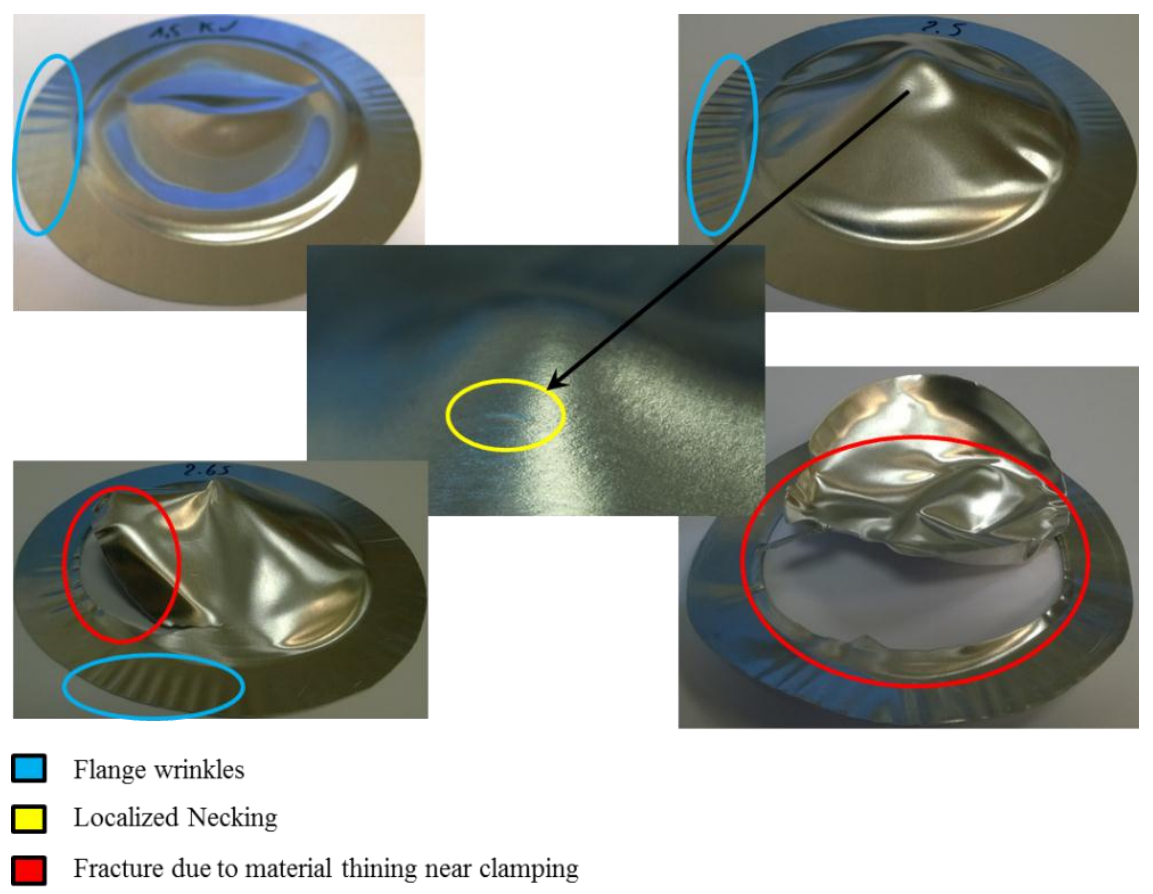

Figure 18 Experimental results of a flat sheep metal expansion. Trails at 1.5kVolts (top-left), 2.5kVolts (top-right), 2.65kVolts (bottom-left) and $3 \mathrm{kVolts}$ (bottom right).

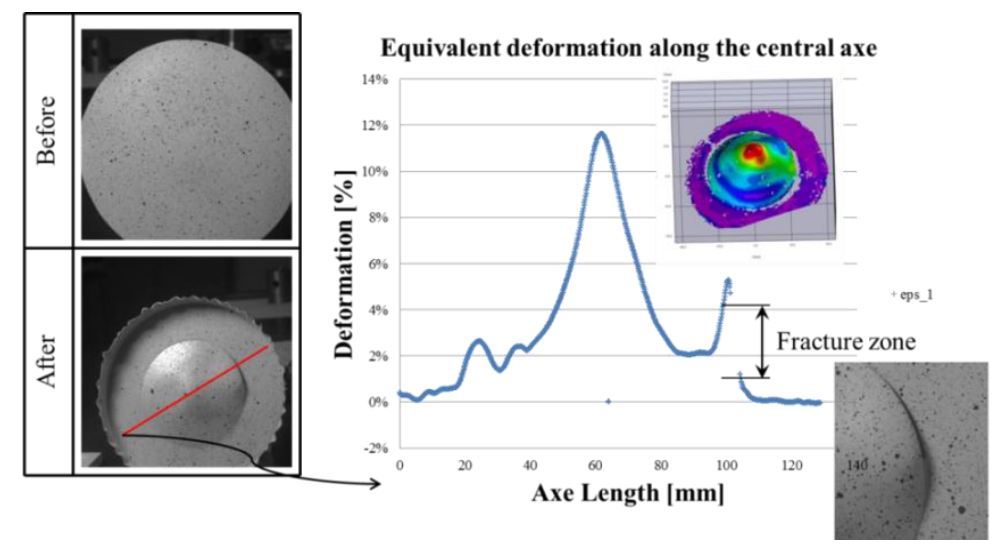

Figure 19 Image analysis facilities using VIC 3D.

To study the formability limit under the given conditions we use the image correlation software VIC3D. At the current moment we can only carry out a post-mortem analysis by comparison of images before and after the magnetic loading. However, this analysis gives access to a full map of deformation field and allows precise identification of the fractured zones. It can be also used as a target map for the deformation field coming from the simulation.

\section{Conclusions}

Understanding of material behavior under high-speed loadings is a quite challenging task that requires careful comprehension of the different phenomena associated: electromagnetic waves propagation, heat transfer and mechanical deformation. For this purpose we have developed a simulation software that allows full interaction between all these aspects to accurately reproduce the physical phenomena. At this stage we have made use of an analytical model to validate the software 
because its simplicity in terms of the mathematical representation, yet its complexity in terms of gathering the main aspects of all the involved physics.

From the chosen study case we have observed how the multidimensional aspects of the simulation will affect the estimation of the different outputs. A reduction of the top head speed of around 15\% is found in comparison with the 1D assumption. The peak force is largely underestimated in the latter one because of the absence of the skin effect within the analysis. This effect is also important for the determination of the temperature distribution, which may be critical for localized softening or phase changes in certain materials.

Numerical simulation has to go hand-by-hand with experiments in order to assess the validity of the results. We have described the main aspects of our experimental settings consisting of a EMF machine with a workbench designed for Direct Free Forming of flat metal sheets.

These experimental settings have allowed us to obtain certain limit values in the application of the EM pulse on the chosen A1050a sheet. It also permits the observation of several phenomena such as wrinkle formation due material flow towards the center; localization of necking at the center of the circular part; Partial and full fracture due to localized thinning near the clamping zone.

\section{Bibliography}

[1] G. Daehn, "High velocity sheet metal forming: state of the art and prognosis for advanced commercialization," Columbus, 2002.

[2] V. Psyk, D. Risch, B. L. Kinsey, a. E. Tekkaya, and M. Kleiner, "Electromagnetic formingA review," Journal of Materials Processing Technology, vol. 211, no. 5, pp. 787-829, May 2011.

[3] V. Psyk, C. Beerwald, A. Henselek, W. Homberg, A. Brosius, and M. Kleiner, "Integration of Electromagnetic Calibration into the Deep Drawing Process of an Industrial Demonstrator Part," Key Engineering Materials, vol. 344, pp. 435-442, 2007.

[4] J. C. Nédélec, “A New Family of Mixed Finite Elements in] R 3," Numer. Math, vol. 50, pp. 57-81, 1986.

[5] R. H. Wagoner and J.-L. Chenot, Metal Forming Analysis. Cambridge University Press, 2001, p. 376.

[6] C. Pelissou, "Discrétisation spatio-temporelle du problème thermique à deux champs. Application au procédé de forgeage à chaud,” MINES ParisTech - CEMEF, 2005.

[7] G. Johnson and W. Cook, "A constitutive model and data for metals subjected to large strains, high strain rated and high temperatures," in Proceedings of the 7th international symposium on ballistics, 1983, pp. 541-547.

[8] G. Fenton, "Development of numerical tools to model plasticity in aluminum due to electromagnetic forces," The Ohio State University, 1996. 